\title{
Use of UAV images to assess narrow brown leaf spot severity in rice
}

\author{
Ning Cai ${ }^{1}$, Xingen Zhou ${ }^{2}$, Yubin Yang ${ }^{2}$, Jing Wang ${ }^{2}$, Dongyan Zhang ${ }^{1 *}$, Rongjie $\mathrm{Hu}^{1}$ \\ (1. Engineering Research Center for Agro-Ecological Big Data Analysis \& Application, Anhui University, Hefei 230601, China; \\ 2. Texas A \&M University System, AgriLife Research and Extension Center, Beaumont, TX 77713, USA)
}

\begin{abstract}
Unmanned aerial vehicle (UAV) remote sensing is a potential tool to reduce crop yield losses caused by numerous diseases through near real-time detection and monitoring on disease progression. However, limited research has been conducted to effectively integrate this technology into current crop management systems for disease control. In this study, the feasibility of assessing the severity of narrow brown leaf spot (NBLS) in rice based on UAV remote sensing platform was explored. RGB and NIR images were obtained using Sentera Multispectral Double 4K sensor attached to DJI INSPIRE 2 drone flying at two altitudes (10 m and $15 \mathrm{~m})$. Ground-truth data on disease severity were collected through visual assessment of field plots with different levels of disease severity. Five out of 21 vegetation indices have a coefficient of determination $\left(R^{2}\right)$ value greater than 0.8 based on unitary linear regression. The index with the highest $R^{2}$ is Excess Green minus Excess Red (ExGR). The results of unitary regression analysis demonstrated more suitability of using RGB images for rice NBLS assessment over NIR images. Further analyses were conducted on disease-infected plot data that were divided into two groups with 2/3 of the plot data as modeling set and the remaining as evaluation set. The ExGR has the highest $R^{2}$ value and the lowest RMSE value in both modeling and evaluation sets regardless of drone flight height (10 $\mathrm{m}$ or $15 \mathrm{~m}$ ). The RMSE at $15 \mathrm{~m}$ is lower than at $10 \mathrm{~m}$ but there was no significant difference of $R^{2}$, thus the $15-\mathrm{m}$ flight height is better than the 10-m height in detecting the levels of disease severity. The comparison of ExGR and HIS-H demonstrated that vegetation index is more suitability for detecting rice NBLS disease with more spectral information. When disease severity data were divided into two score groups ( 0 to 5 and 6 to 9 for the low and high levels of disease, respectively) or three score groups (0 to 3, 4 to 6 and 7 to 9 for the low, moderate and high levels of disease, respectively), the ExGR was more suitable for the detection of the high levels of disease. These results demonstrated the feasibility of using UAV images as a potential tool to assess the severity of NBLS, an important fungal foliar disease in rice worldwide.
\end{abstract}

Keywords: UAV remote sensing, multispectral sensor, narrow brown leaf spot, disease severity, rice DOI: $10.33440 /$ j.ijpaa.20190202.47.

Citation: Cai N, Zhou X G, Yang Y B, Wang J, Zhang D Y, Hu R J. Use of UAV images to assess narrow brown leaf spot severity in rice. Int J Precis Agric Aviat, 2019; 2(2): 38-42.

\section{Introduction}

Rice is a staple food for more than half of the world's population; it plays a critical role in global food security ${ }^{[1]}$. However, rice diseases pose a major threat to rice production and can cause significant economic yield and quality losses each year ${ }^{[2]}$. Narrow Brown Leaf Spot (NBLS) caused by Cercospora janseana is one of the diseases limiting rice production ${ }^{[2]}$. NBLS was first discovered in the United States of America in 1906, and has become a common disease on rice in Australia, Asia, Latin America and North America ${ }^{[3]}$. In recent years, the damage caused by the disease is on the rise ${ }^{[4]}$. It is vital to develop effective prevention and control programs to minimize the damage caused by the NBLS disease. Accurate, real-time monitoring of the initiation and development of NBLS is the first step towards that goal. Traditionally, scouting for the presence of diseases and

\section{Received date: 2019-12-01 Accepted date: 2019- 12-17}

Biographies: Ning Cai, Master student, research interests: computer vision technology, Email: 1042507732@qq.com; Xingen Zhou, research interests: epidemiology and management of rice diseases, Email:xzhou@aesrg.tamu.edu; Yubin Yang, research interests: systems analysis, and agricultural decision systems, Email:yyang@aesrg.tamu.edu; Jing Wang, research interests: software engineering and database management, Email: jingw@aesrg.tamu.edu; Rongjie Hu, research interests: computer vision technology, Email: 1258384661@qq.com.

* Corresponding author: Dongyan Zhang, research interest: computer vision technology, Email: zhangdy@ahu.edu.cn. assessment of disease incidence and severity are carried out by producers or crop consultants. However, this process is very time-consuming and error-prone due to very limited areas of the field that can be covered by scouting ${ }^{[5]}$. Currently, there has been an increasing trend of using non-imaging and imaging spectrum for disease detection and assessment. Graeff et $\mathrm{al}^{[6]}$ used non-imaging spectral information to study the correlation between wheat stripe rust, total lesion and spectral information, and to screen spectral bands that are sensitive to rust lesions. $\mathrm{Li}$ et $\mathrm{al}^{[7]}$ combined principal component analysis (PCA) and probabilistic neural network (PNN) to identify rice stem nematode and rice leaf roller with an accuracy of $96 \%$. Huang et $\mathrm{al}^{[8]}$ established a model based on the first- and second-order differential spectrum to predict the probability of Sclerotinia sclerotiorum infection in celery with the partial least squares regression. Yang et $\mathrm{al}^{[9]}$ selected green normalized difference vegetation index (GNDVI) and soil adjusted vegetation index(SAVI) to monitor rice diseases based on imaging spectrum. Huang et $\mathrm{al}^{[10]}$ established a model by combining aerial hyperspectral images with regression analysis, and retrieved the severity of wheat stripe rust. Delwiche et $\mathrm{al}^{[11]}$ found that hyperspectral images are suitable for detecting wheat scab (FusaHum graminearum Sehw.). Yang et $\mathrm{al}^{[12]}$ compared the difference between multispectral and hyperspectral images in the extraction of cotton root rot, and found that multispectral images are also suitable for large-scale disease monitoring. Jonas and Menz ${ }^{[13]}$ was able to detect wheat powdery mildew and stripe rust based on QuickBird satellite images using a spectral angle mapping 
and hybrid filtering algorithm. Compared with satellite remote sensing, airplane and ground-based sensing systems ${ }^{[14,15]}$, UAV (Unmanned aerial vehicle) has the advantage of low cost, portability, convenient operation, good timeliness and high flexibility ${ }^{[16-18]}$. UAV platforms have been widely used for crop growth monitoring and evaluation ${ }^{[19]}$. However, the evaluation of rice diseases based on UAV is still in its infancy. Zhang et al researched rice sheath blight (Rhizoctonia solani) using images obtained from a multispectral sensor attached to a DJI Phantom 3 drone. The authors combined measured ground-truth NDVI and actual disease data on the ground ${ }^{[20]}$. Although cultivating disease-resistant varieties of rice is the best method for reducing losses, chemical control is most effective when diseases occur. Recently, effective fungicides for control of NBLS have been identified $^{[21,22]}$.

In this study, we explored the feasibility of using the UAV platform to evaluate the severity of rice NBLS at different flight heights. A Sentera (https://sentera.com/) double 4K multispectral sensor was attached to a DJI Inspire 2 drone to capture RGB and NIR images in the field. At the same time, ground truth disease severity data was collected by a plant pathologist, and the prediction model was established based on several indices with the best correlation.

\section{Materials and methods}

\subsection{Experimental design}

The experiment site was located at the Texas A\&M AgriLife Research Center $\left(30.060298^{\circ} \mathrm{N}\right.$ and $\left.94.293370^{\circ} \mathrm{W}\right)$ in Beaumont, Texas, USA. Rice was planted on May 1, 2019, with a total of 40 plots (Figure 1). The NBLS disease severity was rated by visual inspection of each plot, using a scale of $0-9$, with 0 indicating no disease and 9 meaning greatest severity. The numbers next to the arrow indicate plot IDs. Different colors correspond the different levels of disease severity (0 to 9).

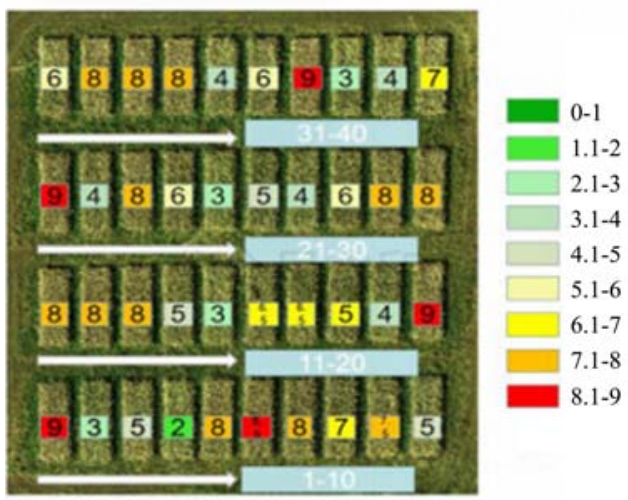

Figure 1 Study plots and disease severity

\subsection{UAV platform and data acquisition}

The 4-motor DJI INSPIRE 2 drone (Figure 2a) has a net weight of $3440 \mathrm{~g}$, a maximum takeoff weight of $4250 \mathrm{~g}$, a GPS hovering accuracy of $0.5 \mathrm{~m} \pm 0.1 \mathrm{~m}$ in the vertical direction and $1.5 \mathrm{~m} \pm 0.3 \mathrm{~m}$ in the horizontal direction. The Sentera Multispectral Double 4K sensor (Sentera, USA) (Figure 2b) offers five spectral bands of blue, green, red, red edge, and NIR, and is capable of capturing 12.3 MP still images. Images were captured from 2:00 to 3:00 PM on Sep. $6^{\text {th }}$, 2019 at two different flight heights of 10 and $15 \mathrm{~m}$ aboveground; the weather was clear with very little wind.

\subsection{Data processing}

Image preprocessing (image mosaic, radiation correction, and band coincidence) was performed using Photoscan 1.4.1 and Envi 5.3 software. The target area was divided into batches with ENVI's vector cutting tool.

Results for image preprocessing are shown in Figure 3. Since plots $9,10,31$ and 32 at $10 \mathrm{~m}$ flight height had incomplete image coverage (Figure 3c and Figure 3d), they were excluded from subsequent analysis for both $10 \mathrm{~m}$ and $15 \mathrm{~m}$ flight height data.

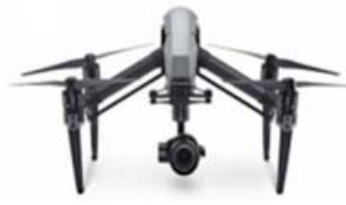

a. DJI INSPIRE 2

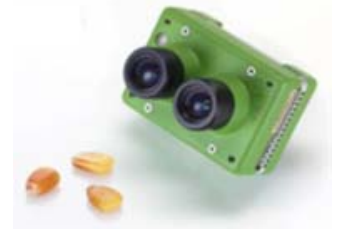

b. Sentera Multispectral Double 4K sensor Figure 2 UAV and camera

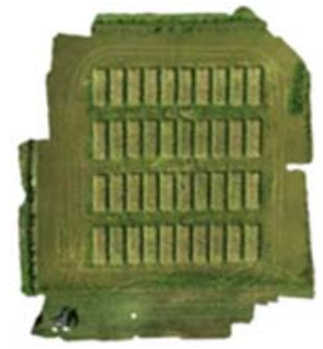

a. $15 \mathrm{~m}$ RGB image

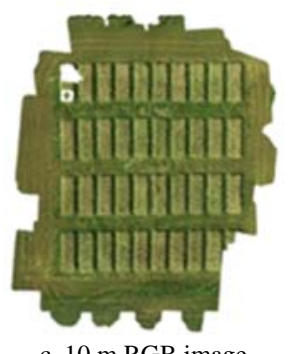

c. $10 \mathrm{~m}$ RGB image

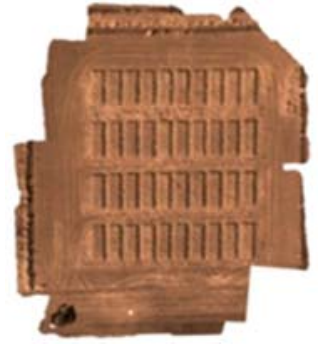

b. $15 \mathrm{~m}$ NIR image

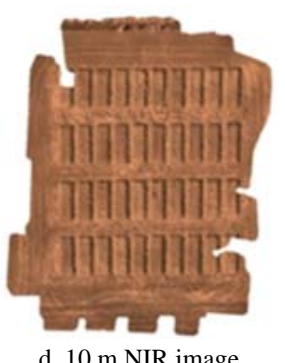

d. $10 \mathrm{~m}$ NIR image
Figure 3 RGB and NIR images of two flight heights

\subsection{Research methods}

Based on ENVI mass cut images, different vegetation indices (Vis) and color space HSI, HSV, HSL and YCbCr were used to evaluate their ability to detect differences in the severity of the NBLS disease at different flight heights (Table 1). We determined the most appropriate vegetation index, color space based on unitary linear regression, and established the inversion model of rice NBLS with good correlation. The precision was validated by the $R^{2}$ (coefficient of determination) and root mean squares error (RMSE).

\section{Results and discussion}

\subsection{Correlations comparison of Vis/color features calculated from RGB and NIR images}

The progression of the NBLS disease in rice causes changes in plant tissue color, and thus different disease severity levels of crops can be detected by digital imaging technique. However, how disease severity is correlated with RGB and NIR spectrum still needs to be established based on scientific experiments and corresponding data analysis. In this study, the correlations between disease severity and Vis/color features calculated from RGB and NIR images were obtained, as shown in Tables 2 and 3 to illustrate which image data were more suitable for differentiating different levels of rice NBLS. Five out of the 21 models of RGB image had an $R^{2}$ value 0.80 or greater, which were the ExGR, 
NGRDI, RGRI, VDVI, HSI-H, respectively (Table 2). The index EXGR at $15 \mathrm{~m}$ flight height was the best, with $R^{2}$ value of 0.89 and RMSE value of 0.70 , followed by VDVI with an $R^{2}$ of 0.8429 and RMSE of 0.8334 . Similar results were observed at $10 \mathrm{~m}$ flight altitude. For the NIR image (Table 3), the index DVI at $15 \mathrm{~m}$ flight height had the highest $R^{2}(0.4097)$ and the lowest RMSE (1.6156), followed by NIR-Red Edge with an $R^{2}$ of 0.3950 and RMSE of 1.6851 . At $10 \mathrm{~m}$ flight height, the $R^{2}$ value of NIR-Red Edge was the 0.3743 with RMSE=1.6634, and followed by DVI $\left(R^{2}=0.3704\right.$, RMSE $\left.=1.6686\right)$. Through the above correlation analysis, it is demonstrated that results of RGB image are better than those of NIR image, and the correlations of visible light Vis are higher than those color space transformations. Moreover, there is no significant difference between two flight heights, only $R^{2}$ value of the correlation at $15 \mathrm{~m}$ is a little better than those of $10 \mathrm{~m}$ flight altitude. These results suggest that indices that incorporate more spectral band (three bands) contain abundant information for more effective disease detection, thus the Vis calculated from RGB/NIR images have higher correlation than single band. However, the results of NIR image are lower than RGB image, this may be due to lack of differentiation in NIR bands to effectively separate different NBLS severities, but additional studies are needed to verify these differences. Data were only collected from two flight heights (10 and $15 \mathrm{~m}$ ), future experiments should expand the flight height to 5 and $20 \mathrm{~m}$ to further explore the effectiveness of disease detection.

\subsection{Modeling and validation of rice NBLS based on Vis and} color features

Results from Tables 2 and 3 suggests that there are better correlations between Vis/color features and disease severity for the RGB image. The coefficients of determination $R^{2}$ of ExGR, NGRDI, RGRI, VDVI, HSI-H are above 0.8 and higher than that of calculated from NIR image. Thus, we chose these Vis/color features to establish the models of disease detection, and analyzed which feature is best for rice NBLS identification. Data from the disease-infected plots were divided into two groups with the ratio of 3:1; data from 27 plots were used as training sets and data from the remaining nine plots as test sample sets.

Table 1 Vegetation indices calculated from NIR and RGB images

\begin{tabular}{|c|c|}
\hline Vis & Formulas \\
\hline \multirow{7}{*}{$\begin{array}{c}\text { NIR } \\
\text { indices }\end{array}$} & Difference Vegetation Index (DVI) $=$ NIR-R \\
\hline & $\begin{array}{l}\text { Normalized Difference Vegetation Index }(\mathrm{NDVI})=(\mathrm{NIR}-\mathrm{R}) / \\
(\mathrm{NIR}+\mathrm{R})\end{array}$ \\
\hline & Ratio Vegetation Index $(\mathrm{RVI})=\mathrm{NIR} / \mathrm{R}$ \\
\hline & Transform Vegetation Index $(\mathrm{TVI})=((\mathrm{NIR}-\mathrm{R}) /(\mathrm{NIR}+\mathrm{R})+0.5)^{1 / 2}$ \\
\hline & NIR - Red Edge \\
\hline & $\mathrm{VI}_{840}=($ NIR - Red Edge $) /($ NIR + Red Edge $)$ \\
\hline & $\mathrm{VI}_{720}=($ Red Edge $-\mathrm{R}) /($ Red Edge $+\mathrm{R})$ \\
\hline \multirow{9}{*}{$\begin{array}{l}\text { RGB } \\
\text { indices }\end{array}$} & $\mathrm{R}^{*}=\mathrm{R} /(\mathrm{R}+\mathrm{G}+\mathrm{B})$ \\
\hline & $\mathrm{G}^{*}=\mathrm{G} /(\mathrm{R}+\mathrm{G}+\mathrm{B})$ \\
\hline & $B^{*}=B /(R+G+B)$ \\
\hline & Excess Green $(E x G)=2 G^{*}-R^{*}-B^{*}$ \\
\hline & Excess Red $(E x R)=1.4 R^{*}-G^{*}$ \\
\hline & ExGR=ExG-ExR \\
\hline & $\begin{array}{l}\text { Normalized Green-Blue Difference Index }(\mathrm{NGRDI})==(\mathrm{G}-\mathrm{R}) / \\
(\mathrm{G}+\mathrm{R})\end{array}$ \\
\hline & Red-Green Ratio Index (RGRI)=R/G \\
\hline & $\begin{array}{l}\text { Visible-band Difference Vegetation Index }(\mathrm{VDVI})=(2 \mathrm{G}-\mathrm{R}-\mathrm{B}) / \\
(2 \mathrm{G}+\mathrm{R}+\mathrm{B})\end{array}$ \\
\hline
\end{tabular}

Table 2 Correlations between disease severity and Vis/color features calculated from RGB image

\begin{tabular}{|c|c|c|c|c|}
\hline \multirow{2}{*}{$\begin{array}{l}\text { Vis/color } \\
\text { features }\end{array}$} & \multicolumn{2}{|c|}{$15 \mathrm{~m}$ flight height } & \multicolumn{2}{|c|}{$10 \mathrm{~m}$ flight height } \\
\hline & $R^{2}$ & RMSE & $R^{2}$ & RMSE \\
\hline ExG & 0.8343 & 0.8562 & 0.7595 & 1.0312 \\
\hline ExGR & 0.8904 & 0.6965 & 0.8900 & 0.6976 \\
\hline ExR & 0.5849 & 1.3548 & 0.5711 & 1.3771 \\
\hline NGRDI & 0.8319 & 0.8620 & 0.8495 & 0.8155 \\
\hline RGRI & 0.8305 & 0.8657 & 0.8433 & 0.8323 \\
\hline VDVI & 0.8429 & 0.8334 & 0.8601 & 0.7866 \\
\hline HSI-H & 0.8292 & 0.869 & 0.8435 & 0.8321 \\
\hline HSI-S & 0.3605 & 1.6816 & 0.4002 & 1.6286 \\
\hline HSI-I & 0.0419 & 2.0584 & 0.0181 & 2.0837 \\
\hline HSL-H & 0.7777 & 0.9913 & 0.7885 & 0.9688 \\
\hline HSL-S & 0.7206 & 1.0711 & 0.7233 & 1.0252 \\
\hline HSL-L & 0.0677 & 17.521 & 0.0270 & 2.0743 \\
\hline HSV-H & 0.7704 & 1.0078 & 0.8411 & 0.8385 \\
\hline HSV-S & 0.4654 & 1.5375 & 0.5382 & 1.5165 \\
\hline HSV-V & 0.0210 & 2.0953 & 0.0028 & 2.0999 \\
\hline YCbCr-Y & 0.0187 & 2.0831 & 0.0037 & 2.0989 \\
\hline YCbCr-Cb & 0.0673 & 2.0308 & 0.0694 & 2.0285 \\
\hline YCbCr-Cr & 0.8285 & 0.8708 & 0.7858 & 0.9735 \\
\hline $\mathrm{R}^{*}$ & 0.2409 & 1.8322 & 0.2154 & 1.8626 \\
\hline $\mathrm{G}^{*}$ & 0.0861 & 2.0102 & 0.1603 & 1.9269 \\
\hline $\mathrm{B}^{*}$ & 0.1718 & 1.9137 & 0.1718 & 1.9150 \\
\hline
\end{tabular}

Table 3 Correlations between disease severity and Vis calculated from NIR image

\begin{tabular}{cccccc}
\hline \multirow{2}{*}{$\begin{array}{c}\text { Vegetation } \\
\text { indexes }\end{array}$} & \multicolumn{2}{c}{$15 \mathrm{~m}$ flight height } & & \multicolumn{2}{c}{$10 \mathrm{~m}$ flight height } \\
\cline { 2 - 3 } \cline { 5 - 6 } & $R^{2}$ & RMSE & $R^{2}$ & RMSE \\
\hline DVI & 0.4097 & 1.6156 & & 0.3704 & 1.6686 \\
NDVI & 0.0009 & 2.1019 & & 0.1518 & 1.9637 \\
RVI & 0.0000 & 2.1028 & 0.1651 & 1.9215 \\
TVI & 0.0011 & 2.1016 & 0.1756 & 1.9094 \\
NIR-Red Edge & 0.3950 & 1.6851 & 0.3743 & 1.6634 \\
VI840 & 0.0494 & 2.0503 & 0.0210 & 2.0806 \\
VI720 & 0.1678 & 1.9183 & 0.1718 & 1.9150 \\
\hline
\end{tabular}

The modeling and verification results at two flight heights of $15 \mathrm{~m}$ and $10 \mathrm{~m}$ were showed in Table 4. ExGR has the highest determination coefficient $R^{2}$ and the lowest RMSE value in both modeling and verification results regardless of flight height $(10 \mathrm{~m}$ or $15 \mathrm{~m}$ ). At $15 \mathrm{~m}$ flight height, the modeling $\mathrm{R}^{2}$ value of ExGR reaches 0.9494 with RMSE equals the 0.5864, while the verification $R^{2}$ value reaches 0.9397 with $\mathrm{RMSE}=0.8915$. At $10 \mathrm{~m}$ flight height, the $R^{2}$ value of modeling is the 0.9561 with RMSE $=0.6288$, the verification results has an $R^{2}$ of 0.9312 and RMSE of 0.9991. Although there is no significant difference for coefficient of determination $R^{2}$ between two flight heights, the RMSE value at $15 \mathrm{~m}$ flight height is lower than the result of $10 \mathrm{~m}$. Therefore, $15 \mathrm{~m}$ flight height is more suitable for disease detection, with additional saving in flight time. Moreover, we also found that color feature HIS-H of single band has a $R^{2}$ value of 0.9066 at $15 \mathrm{~m}$ flight height, and it is lower than that of ExGR of two bands. The results were similar to those at $10 \mathrm{~m}$ flight height. Therefore, it can be concluded that that indices with more spectral information are more effective to identify the rice NBLS. 
Table 4 Modeling and validation of rice NBLS

\begin{tabular}{|c|c|c|c|c|c|c|c|c|}
\hline \multirow{3}{*}{ Vis/color features } & \multicolumn{4}{|c|}{ Modeling results } & \multicolumn{4}{|c|}{ Verification results } \\
\hline & \multicolumn{2}{|c|}{$R^{2}$} & \multicolumn{2}{|c|}{ RMSE } & \multicolumn{2}{|c|}{$R^{2}$} & \multicolumn{2}{|c|}{ RMSE } \\
\hline & $15 \mathrm{~m}$ & $10 \mathrm{~m}$ & $15 \mathrm{~m}$ & $10 \mathrm{~m}$ & $15 \mathrm{~m}$ & $10 \mathrm{~m}$ & $15 \mathrm{~m}$ & $10 \mathrm{~m}$ \\
\hline ExGR & 0.9494 & 0.9561 & 0.5864 & 0.6288 & 0.9397 & 0.9312 & 0.8915 & 0.9991 \\
\hline NGRDI & 0.9154 & 0.9290 & 0.7407 & 0.8194 & 0.9184 & 0.9167 & 0.9906 & 1.0306 \\
\hline RGRI & 0.9126 & 0.9290 & 0.7409 & 0.8183 & 0.9152 & 0.9113 & 1.0060 & 1.0938 \\
\hline VDVI & 0.9209 & 0.9357 & 0.7061 & 0.7801 & 0.9207 & 0.9170 & 0.9933 & 1.0052 \\
\hline HIS-H & 0.9066 & 0.9278 & 0.7469 & 0.8448 & 0.9356 & 0.9104 & 0.9664 & 1.0746 \\
\hline
\end{tabular}

\subsection{Modeling analysis under different disease severity grading}

The above analysis identified the ExGR as the best index for disease severity detection. To determine the effectiveness of the ExGR in detecting rice NBLS with a different disease severity classification scheme, we divided disease severity data into two groups ( $0 \sim 5$ and $6 \sim 9$ for the low and high levels of disease severity, respectively) and three groups (0 3, 4 6 and 7 9 for the low, moderate and high levels of disease severity, respectively). As shown in Table 5, regardless of the number of disease severity groupings, the $R^{2}$ of the ExGR are always higher than other vegetation indexes and color feature. In the two-grade grouping scheme, $R^{2}$ changed from 0.8191 to 0.9209 , while $R^{2}$ changed from $0.7261,0.8847$ to 0.9242 for the three-grade grouping scheme. The results indicate that it is more effective to detect the high level of disease severity than the low level of disease severity. Therefore, the ExGR is more suitable for the detection of high levels of disease infection.

Table 5 Coefficients of determination with different disease classification schemes

\begin{tabular}{lcccccc}
\hline & \multicolumn{5}{c}{ Disease severity classification } \\
\cline { 2 - 7 } Vis/color feature & \multicolumn{2}{c}{ Two grades } & \multicolumn{3}{c}{ Three grades } \\
\cline { 2 - 7 } \cline { 5 - 7 } & $0 \sim 5$ & $6 \sim 9$ & $0 \sim 3$ & $4 \sim 6$ & $7 \sim 9$ \\
\hline ExGR & 0.8191 & 0.9209 & 0.7261 & 0.8847 & 0.9242 \\
NGRDI & 0.7632 & 0.9147 & 0.6500 & 0.8503 & 0.9208 \\
RGRI & $R^{2}(15 \mathrm{~m})$ & 0.7629 & 0.9123 & 0.6450 & 0.8516 & 0.9197 \\
VDVI & 0.7450 & 0.9230 & 0.6072 & 0.8448 & 0.9338 \\
HIS-H & 0.7408 & 0.9163 & 0.6234 & 0.8308 & 0.9284 \\
\hline
\end{tabular}

\section{Conclusions}

In this study, UAV-based RGB and NIR images were obtained at two different heights of $10 \mathrm{~m}$ and $15 \mathrm{~m}$ over an experimental field with 36 research plots. Ground truth assessment of disease severity was obtained on the same day. Results of unitary linear regression were quantitatively evaluated. Five inversion models of RGB image (ExGR, NGRDI, RGRI, VDVI, HSI-H) show higher $R^{2}$ values and low RMSE values. The best model is ExGR with an $R^{2}$ of 0.8904 , and RMSE of 0.6965 at $15 \mathrm{~m}$ flight altitude. Among the results of unitary linear regression based on NIR image, DVI is the best, and the highest $\mathrm{R}^{2}$ at two flight altitudes is 0.4097 . The correlation analysis above showed more suitability of RGB image for detecting rice NBLS disease, and the correlations of visible light Vis are higher than those color space transformations. Moreover, comparisons of two heights showed there was no significant difference between two flight heights. Furthermore, the modeling and verification results showed the ExGR has the highest determination coefficient $R^{2}$ and the lowest RMSE value. Comparing RMSE between two heights, we conclude that $15 \mathrm{~m}$ flight height is more suitable for detecting the rice NBLS disease. Finally, different disease severity level classifications suggested the ExGR is more suitable for the detection of high levels of disease infection. In the future studies, flight plans should be optimized with more levels of flight height, more vegetation indexes, color spaces and other features to improve the inversion model, which may provide guidance for disease prevention and control.

\section{Acknowledgments}

We greatly thank Mr. Pat Carre and Mr. Leon Holgate for operating with the UAV and Drs. Lloyd T. Wilson, Fugen Dou and Stanley Samonte for contributing the purchase of the devices used in this study. This research was funded by National Natural Science Foundation of China (Grant No. 41771463 and 41771469), Anhui Provincial Major Science and Technology Projects (Grant No.18030701209).

\section{[References]}

[1] Muthayya S, Sugimoto J D, Montgomery S. An overview of global rice production, supply, trade, and consumption. Annals of the New York Academy of Sciences, 2014, 1327(1): 7. doi: 10.1111/nyas.12540

[2] Zhou X G, Young-Ki J. Disease management. The Texas rice production guidelines. Texas AgriLife Research and Texas AgriLife Extension. B-6131. 2014, pp. 44-56.

[3] Kirandeep K M, Clayton A. Hollier, Donald E G. Effect of planting date, fungicide timing and cultivar susceptibility on severity of narrow brown leaf spot and yield of rice. Crop Protection, 2016, 90: 186-187. doi: 10.1016/j.cropro.2016.07.029

[4] Peng S B, Tang Q Y, Zou Y B. Current status and challenges of rice production in China. Plant Production Science, 2009, 12(1): 3-4. doi: 10.1626/pps.12.3

[5] Zhang J C, Yuan L, Wang J H. Research progress of crop diseases and pests monitoring based on remote sensing. Transactions of the Chinese Society of Agricultural Engineer, 2012, 28(20): 1. doi: 10.3969/ j.issn.1002-6819.2012.20.001.

[6] Graeff S, Link J, Claupein. Identification of powdery mildew (Erysiphegraminis sp. tritici) and take-all disease (Gaeumannomyces graminis sp. tritici) in wheat (Triticum aestivum L.) by means of leaf reflectance measurements. Central European Journal of Biology, 2006, 1(2): 275-288. doi: 10.2478/s11535-006-0020-8

[7] Li B, Liu Z Y, Huang J F. Hyperspectral identification of rice diseases and pests based on principal component analysis and probabilistic neural network. Transactions of the Chinese Society of Agricultural Engineer, 2009, 25(9): 143-146. doi: 10.3969/j.issn.1002-6819.2009.09.026

[8] Huang, J F, Apan, A. Detection of sclerotinia rot disease on celery using hyperspectral data and partial least squares regression. Journal of Spatial science, 2006, 52(2): 129-142. doi: 10.1080/14498596.2006.9635087

[9] Yang C M, Cheng C H, Chen R K. Changes in spectral characteristices of rice canopy infested with brown planthopper and leaffolder, Crop Science, 2007, 47(1): 329-335. doi: 10.2135/cropsci2006.05.0335

[10] HUANG W J, Lamb D W, Niu Z. Identification of yellow rust in wheat using in-situ spectral reflectance measurements and airborne hyperspectral imaging. Precision Agriculture, 2007, 8(5): 187-197. doi: 10.1007/ s11119-007-9038-9

[11] Delwiche S R, Kim M S. Hyperspectral imaging for detection of scab in wheat. Biological Quality and Precision Agriculture II, 2000, 4203: 
13-20. doi: 10.1117/12.411752

[12] Yang C H, Everitt J H, Fernandes C J. Comparision of airborne multispectral and hyperspectral imagery for mapping cotton root rot. Biosystems Engineering, 2010, 107(2): 131-139. doi: 10.1016/ j.biosyste- mseng.2010.07.011

[13] Jonas F, Menz G. Multi-temporal wheat disease detection by multispectral remote sensing. Precision Agriculture, 2007, 8(3): 161-172. doi: 10.1007/s11119-007-9036-y

[14] Zhao C J. The development of agricultural remote sensing research and application. Journal of Agricultural Machinery, 2014, 45(12): 277-293.. doi: 10.6041/ j.issn.1000-1298.2014.12.041

[15] Li D R, Li M. Research progress and application prospect of UAV remote sensing system. Journal of Wuhan University (Information Science Edition), 2014, 39(05): 505-513+540. doi: 10.13203/ j.whugis2014 0045

[16] Mahlein A K. Plant disease detection by imaging sensors-parallels and specific demands for precision agriculture and plant phenotyping. Plant Disease, 2016, 100(2): 241. doi: 10.1094/PDIS-03-15-0340-FE

[17] Ballesteros R, Ortega J F, Hernandez D. Applications of georeferenced high-resolution images obtained with unmanned aerial vehicles. Part I : Description of image Acquisition and processing. Precision Agriculture, 2014, 15(6): 579. doi: 10.1007/s11119-014-9355-8

[18] Zhang J, Yang C H, Song H B. Evaluation of an airborne remote sensing platform consisting of two Consumer-Grade cameras for crop identification. Remote Sensing, 2016, 8(3): 257. doi: 10.3390/rs8030257

[19] Mulla, David J. Twenty five years of remote sensing in precision agriculture: Key advances and remaining knowledge gaps. Biosystems Engineers, 2013, 114(4): 358-371. doi: 10.1016/j.biosystemseng.2012.08.009

[20] Zhang D, Zhou X G, Zhang J, Lan Y, Xu C, Liang D. Detection of rice sheath blight using an unmanned aerial system with high-resolution color and multispectral imaging. PLoS ONE, 2018, 13(5): e0187470. doi: 10.1371/journal.pone.0187470

[21] Uppala S, Zhou X G. Field efficacy of fungicides for management of sheath blight and narrow brown leaf spot of rice. Crop Protection, 2018, 104: 72-77. doi: 10.1016/j.cropro.2017.10.017

[22] Uppala S, Zhou X G. Optimum timing of propiconazole to manage narrow brown leaf spot in the main and ratoon crops in Texas. Crop Protection, 2019, 124: 1-6. doi: 10.1016/j.cropro.2019.104854 